\title{
Impact of increased renewables on natural gas markets in eastern United States
}

\author{
Neha NANDAKUMAR ${ }^{1}$, , Anuradha M. ANNASWAMY $^{2}$
}

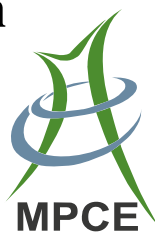

\begin{abstract}
This paper explores the market structures of natural gas and electricity as well as the interdependence of natural gas prices and bids with increasing reliance on natural gas as the penetration of renewable energy resources increases in order to complement their intermittencies. In particular, the paper will attempt to answer the following two questions: What could the generation mix look like in 2030 with a renewable-rich generation landscape and how could this impact gas prices? How do gasfired generator (GFG) generation volatility, their prices, and their bids for gas change between 2015 and 2030 with increased penetration of renewables? In order to answer these questions, computational models are derived using forecasting and regression analysis tools and an auction model.
\end{abstract}

Keywords Natural gas markets, Gas-fired generators, Regression analysis, Optimal auction model, Volatility, Gas prices

CrossCheck date: 10 April 2017

Received: 7 January 2017/Accepted: 10 April 2017/Published online: 4 May 2017

(C) The Author(s) 2017. This article is an open access publication

\section{Neha NANDAKUMAR \\ nehan@mit.edu}

Anuradha M. ANNASWAMY

aanna@mit.edu

1 Institute for Data, Systems, and Society, Massachusetts Institute of Technology, Cambridge, MA, USA

2 Department of Mechanical Engineering, Massachusetts Institute of Technology, Cambridge, MA, USA

\section{Introduction}

Global concerns over climate change as well as increasing energy consumption necessitate a cleaner future electric grid. Fossil-fuel based power plants emit tons of carbon dioxide into the atmosphere, a primary gas pollutant in the United States accounting for $82 \%$ of greenhouse gas emissions. Environmental regulations like the EPA's Clean Power Plan aim to combat climate change by proposing carbon dioxide emissions reduction by $30 \%$ by 2030 from power plants. In order to achieve this, states across the country are retiring coal plants and are seeking to replace them with renewable energy resources such as wind and solar power. Since the latter can be highly volatile, increasing their penetration into the power grid calls for quick, cheap, clean, and flexible back-up fuels to ensure reliability. Gas-fired generators (GFGs) are sought after as this reliable back-up fuel due to their flexibility, quick ramp rates, and low cost. As a result, natural gas (NG) and electricity networks are getting increasingly linked and interdependent.

The electricity infrastructure consists of generators from which power is transferred via long distance, high-voltage transmission lines, with the voltage gradually stepped down through distribution systems to the end-user. With demand largely treated as an uncontrolled, exogenous input, electric utilities have an assumed 'obligation to serve' in which generation needs to be operated to meet this exogenous load demand at all times [1]. This balance between supply and demand is typically carried out by independent system operators. The NG network is quite similar to the electricity network, in terms of the network topology - it consists of transmission (pipelines), producers (wells), storage, and consumers. Pipelines use compressors along the line to create the flow of NG from the injection 
point on the line to the consumer of the NG. NG marketers, facilitate movement of NG by coordinating the sale of gas quantity and pipeline capacity contracts.

Significant differences exist between electricity markets and gas markets in the United States. Though both markets have been deregulated, their structures are quite distinct. In deregulated electricity markets, the supply of electricity is organized through a day-ahead and real-time market, with the former operating on a 24-hour time-scale and the latter clearing anywhere between 5 and 15 minutes depending on the geographical location. At the wholesale level, a centralized balancing authority such as the ISO supervises the operation of these markets. And in each market, the offers made and schedules posted depend significantly on the availability of power generation as well as electricity demand. On the natural gas side, there are capacity markets and commodity markets, for buying NG quantities and buying NG transportation, respectively. Market contracts pertaining to the gas industry can be classified as firm or interruptible. Firm contracts are long term and are typically used by Local Distribution Companies (LDC). Moreover, these contracts incur an additional reservation price which pays off investment in pipeline infrastructure. Interruptible contracts are flexible and typically used by GFGs [2]. Unlike the electricity market, gas markets do not have an ISO but are rather deregulated entirely in which the spot markets consist of bilateral contracts between the gas marketer and consumers (utilities or GFGs obtain gas off this market). Given the above significant differences between the gas and electricity infrastructures, and the growing reliance of the electricity sector on natural gas, it is imperative that the effect of this reliance be explored in a quantitative manner. The focus of this paper is thus to examine how changes in the electricity sector, specifically increased renewable penetration, impact gas market factors such as gas price and volatility in GFG bids for gas. Traditional models of the electricity infrastructure and markets have aimed to maximize net social benefits or minimize costs across an optimized electricity network [3]. Research investigations have examined the changing generation mix including the impact of renewable generation on the electric grid as a whole and wholesale markets in particular. For example, the New England States Committee on Electricity, or NESCOE, explored the impacts of various scenarios of increased renewable capacity on the electric transmission in New England [4]. Econometric models have been explored in $[5,6]$ to determine wind generation impacts on electricity prices and price volatility, mostly examining the merit-order effect that increased wind penetration has on the grid. Reference [7] looks into the relationship between wind power feed-in to the grid and wholesale electricity price. In contrast, the focus of this paper is to examine the growing role of natural gas within the electric grid, especially in light of this increased renewable generation, and how it impacts natural gas markets and in turn wholesale electricity markets in New England.

Of late, several investigations have been launched to explore the coordination between natural gas and electricity in terms of reliable transportation, scheduling, and communication, especially in times of peak demand. Much of this work has explored the possibilities of shortages in gas supply to GFGs due to transportation and pipeline capacities. Some studies have addressed GFGs' potential need for firm contracts and how interruptible ones may prove unreliable in times of peak demand due to weather events, especially the polar vortex [8]. Traditional natural gas modeling has addressed transportation models, investment models, and value chain models (see references in [2]). Transportation models of natural gas analyze the physical properties of the pipelines in a mathematically tractable way and are combined within hybrid models to analyze impacts on electricity power flows. Investment models are used by firms for profit-maximization decisionmaking of whether to invest or not in certain large scale oil and gas power plant projects in different fields [9]. These investment models are very important to analyze due to greater discoveries of shale gas and future uncertainty in production for future consumer demand. Value chain models explore planning and operations of natural gas pipeline and infrastructure, including factors like markets, production, processing, transportation, and contracts; these models are created to capture the liberalization or deregulation of the markets as well, while maintaining a physical pipeline topology $[10,11]$. Some previous work on natural gas markets have explored the idea of the "contracts problem" between producers and pipelines, as well as buyers and sellers, to determine pricing of "take-or-pay" contracts [12], or have explored regulatory gaps and prices with perfect and imperfect competition in the gas markets [13]. Game theory has been used in some applications to explore investments into natural gas [14], as well as to the LNG markets and shipping of LNG [15].

Combined gas-electric models weigh the costs and benefits of all players simultaneously within each individual network when determining the optimal set of natural gas and power flows as well as prices. When studying the interdependency between gas-electric networks, energy security and reliability of the grid has been a focus; in New England, there are two significant supply-related factors that have contributed to shortages and high regional gas (and therefore electricity) prices; first, deliveries of regasified liquefied natural gas (LNG) from Northeast terminals dropped more than $30 \%$ in 2014 compared to the same time period in 2013 [16]. Second, the major interstate and intrastate pipelines that transport natural gas into and 
within New England have been congested, especially during the winter months and polar vortex. An expansion of the pipeline system could alleviate pipeline constraints, but the cost-effectiveness of these pipeline expansion projects (which are passed on to consumers) remains difficult for GFGs and other parties involved [16]. It should be noted however that most of these efforts have been directed towards physical and operational interdependencies and the modeling of the material flow and effects of pipeline congestion rather than the economic flow and its impact on the gas-electricity interdependence. The latter is the focus of this paper, and seeks to examine the impact of the changing grid on natural gas market factors such as gas spot prices, as well as the impact on the GFG-gas marketer transactions.

The goal of this paper is to explore how increased penetration of renewable energy resources impacts gas markets, the role of GFGs in general, and gas prices in particular. Increasing intermittent renewable sources currently brings uncertainty to gas procurement, and increased penetration of renewables will increase this uncertainty even further. Uncertainties do two things: first, any uncertainty in procuring gas increases gas spot prices. Second, uncertainty increases risk for GFG-bids into the gas markets. We quantify these two statements in this paper, which correspond to the two main objectives of this paper. The first objective is addressed via forecasting a future generation mix to determine the impact of increased wind and solar generation on gas generation and gas prices. The impact that increased wind and solar generation has on gas prices is addressed via a regression model. The second objective is the quantification of the risk for GFG-bids with a changing generation mix which is addressed through these econometric models and a first price optimal auction model. We explore within our model how volatility in wind/solar (WS) generation can impact the gas market; based on the results we explore some implications. Based on the results obtained, we make a few recommendations for how GFGs should participate in the gas market.

\section{Forecasting future generation mix and gas price}

The first objective of this paper is to develop a computational model for variations in gas-based power generation and gas price with increasing renewable penetration. To realize this objective, three scenarios of a changing grid for 2030 are analyzed in which average annual renewable penetration is increased to $10 \%, 20 \%$, and $30 \%$ of the total generation mix (as opposed to the current $2.5 \%$ annual average in New England). The major assumptions are that total electricity demand remains constant in each 2030 scenario (given assumptions of increased energy efficiency efforts in MA by 2030), that there is no coal in 2030, and that nuclear generation decreases in New England. Solar and wind generation are forecasted via an Auto-Regressive model [17] for 2030.

A schematic of the methodology that will be adopted to meet the objective of this section is depicted in Fig. 1, where Step 1 of the analysis is to create three different potential scenarios for a future grid in 2030 given forecasts and near future policies that may be in place in New England pertaining to what extent each fuel may or may not participate. To determine how wind/solar generation impacts the rest of the generation mix and gas usage, three scenarios are determined of potential increase in wind/solar generation with varying percentages and how the rest of the generation mix will change as a result. Step 2 includes calculating the daily profiles of each fuel type to come up with a total future generation mix. This is accomplished using two parts, where Step 2a includes modelling and forecasting the wind/solar generation time series, and Step $2 b$ includes calculating the rest of the daily profiles for the remaining fuel types in each of the three 2030 scenarios. Step 3 will include the derivation of a gas price regression model to examine how increased wind and solar generation can impact gas prices.

\subsection{Determining scenarios of changing 2030 generation mix}

To predict scenarios of a future generation mix, it is important to understand what policy changes may occur in the near future and how they will shape the generation mix. Table 1 depicts what the generation mix may look like in 2030, based on various studies for the New England area [18-21]. As previously mentioned, it is assumed that natural gas is the main back-up fuel to renewable generation to maintain power balance because it is the most flexible to

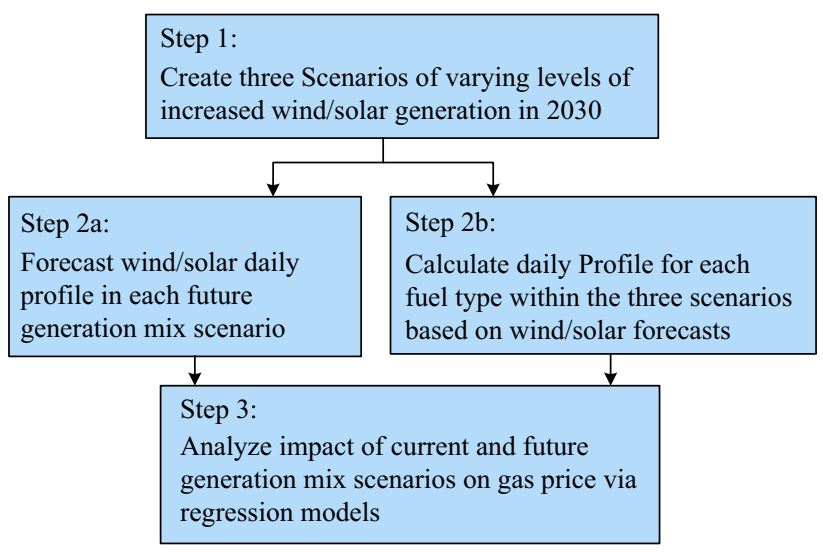

Fig. 1 Overall approach used in objective 1 to determine regression models for gas and electricity prices 
Table 1 Expected growth or decline in generation mix

\begin{tabular}{|c|c|c|}
\hline Fuel & Growth & Decline \\
\hline Wind/solar & $\begin{array}{l}20 \% \text { of generation mix (with } 15 \% \text { being wind, including off-shore } \\
\text { wind). This is part of } 40 \% \text { RPS by } 2030 \text { projected }\end{array}$ & \\
\hline Other renewables & $7 \sim 10 \%$ of mix & \\
\hline Hydro & $10 \sim 30 \%$ of generation mix by 2030 depending on policy & \\
\hline Natural gas & $50 \sim 60 \%$ of generation mix by 2030 depending on policy & \\
\hline Nuclear & & Retirements of $17 \% \sim 48 \%$ by 2030 \\
\hline Oil & May increase slightly in dual fuel capability with gas & \\
\hline Coal & & Expected to be entirely retired by 2030 \\
\hline
\end{tabular}

ramp up and down, and the least pollutant in terms of carbon dioxide emissions into the environment [22].

Three different scenarios of interest are examined below in Table 2. Given that renewable portfolio standards (RPS), an environmental mandate requiring a certain percentage of the resource mix be renewables, may vary from state to state [23] and that wind/solar may contribute up to $30 \%$ of the generation mix by 2030 , three scenarios in which wind/solar generation makes up an average of 10\%, 20\% and $30 \%$ of the grid are considered. The scenarios are depicted in Table 2 below where the percentages represent the average percentage each fuel type makes up within the generation mix in the year 2030. As seen in Table 1 above, coal is expected to be absent by 2030 and is therefore $0 \%$ in all three scenarios. Nuclear generation is expected to decline, and is assumed to therefore be halved by 2030 . Considering that different policy drivers may impact any increases or decreases in hydro, oil and other renewable sources, the rest of the generation profiles in the mix are maintained constant between 2015 and 2030. That is, hydro, oil, and other renewable generation is maintained to encompass on average $7.6 \%, 1.6 \%$, and $6.7 \%$ of the total generation mix in 2030 as it was on average in 2015. Natural gas generation is the only source, besides wind/solar, that changes between the three scenarios. Natural gas is the fuel that makes up any generation gap to meet power demand in the wake of retiring coal and

Table 2 Arrangement of channels

\begin{tabular}{llll}
\hline Channels & Scenario 1 $(\%)$ & Scenario 2 $(\%)$ & Scenario 3 $(\%)$ \\
\hline Wind/solar & 10 & 20 & 30 \\
Gas & 59.2 & 49.2 & 39.2 \\
Coal & 0 & 0 & 0 \\
Nuclear & 14.9 & 14.9 & 14.9 \\
Hydro & 7.6 & 7.6 & 7.6 \\
Oil & 1.6 & 1.6 & 1.6 \\
$\begin{array}{l}\text { Other } \\
\text { renewables }\end{array}$ & 6.7 & 6.7 & 6.7 \\
\hline
\end{tabular}

nuclear plants [21], and therefore is considered to be the flexible fuel that makes up any gaps between power supply and demand. The ramp rates of Natural Gas units are currently of the order of $50 \mathrm{MW} / \mathrm{min}$ and are expected to be even faster as we move towards the next decade [24].

This paper does not address scenarios in which gas consumption itself increases, but rather into how the volatility of gas demand will change should natural gas still remain the marginal fuel and used not just for powering peaking units. It is also assumed that the total electricity demand is the same in 2030 as in 2015 with the daily electricity profile maintained. This is supported by the conclusions of [25] which show that ISO-NE predicts electricity demand to remain the same in the next 10 years due to expected energy efficiency efforts that are being made by all six states in the region. Given the constant demand in electricity, it follows that an increasing wind/solar generation together with reductions in coal and nuclear based generation naturally can allow one to conclude that gas generation itself may decrease. This is because with increasing renewables, the supply stack shifts to the right and higher cost units are getting displaced, leaving the marginal fuel in some cases to be the lower cost gas units. The regression analysis will shed light on the correlation between these factors. Finally, we assume that gas demand profiles (supply to the grid) will stay similar to the current trend, with increased load during cold snaps. We refer the reader to [26] for a deeper investigation of varying gas demand and its elasticity.

\subsection{Determining daily coal, nuclear, natural gas profiles in 2030 scenarios}

In what follows, we denote $X_{t, 2030}^{i}$ as power generation due to asset $i$ in 2030 on day $t$, while $\hat{X}_{t}$ is a quantity that corresponds to a predicted value of $X_{t}$. The annual average generation in the year 2030 for each fuel type was determined for each of the three scenarios. The daily profile, based off of these average generation values, was determined given each fuel type's daily profile of 2015. That is, 
the overall profile of daily points for nuclear in 2030 is calculated as:

$\hat{X}_{t, 2030}^{\text {Nuclear }}=\overline{X_{2030}^{\text {Nuclear }}}+\delta_{1}$

$\delta_{1}=X_{t, 2015}^{\text {Nuclear }}-\overline{X_{2030}^{\text {Nuclear }}}$

where $\hat{X}_{t, 2030}^{\text {Nuclear }}$ and $X_{t, 2015}^{\text {Nuclear }}$ are nuclear generation on day $t$ in 2030 and $2015 ; \overline{X_{2030}^{\text {Nulear }}}$ and $\overline{X_{2015}^{\text {Nuclear }}}$ are the average nuclear generation in 2030 and 2015; $\delta_{1}$ is the corresponding devi-

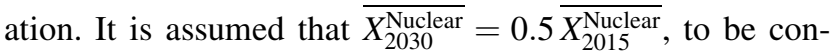
sistent with the assumptions made in Table 1. Such a construction allows one to maintain the overall yearly profile for nuclear generation and inter-day variance/volatility in the future scenario as well.

The only two generation profiles that will change among the three scenarios is gas generation and wind/solar generation, where the gas generation profile is dependent upon all the other profiles as it is the supply to meet any remaining demand on a given day. Therefore, gas generation is determined to be the remainder of any demand that needs to be met on a daily basis, as below.

$$
\begin{aligned}
\hat{X}_{t, 2030}^{\mathrm{Gas}}= & D_{\mathrm{total}, i}-\left(\hat{X}_{t, 2030}^{\mathrm{WS}}+\hat{X}_{t, 2030}^{\mathrm{Nuclear}}+\hat{X}_{t, 2030}^{\mathrm{Coal}}\right. \\
& \left.+\hat{X}_{t, 2030}^{\mathrm{Hydro}}+\hat{X}_{t, 2030}^{\mathrm{Oil}}+\hat{X}_{t, 2030}^{\text {Others }}\right)
\end{aligned}
$$

where $D_{\text {total }, i}$ is the total electricity demand; $\hat{X}_{t, 2030}^{\mathrm{WS}}$ is the predicted wind/solar power generation in 2030 for any the three scenarios described in Table $2 ; \hat{X}_{t, 2030}^{\mathrm{Hydro}}, \hat{X}_{t, 2030}^{\mathrm{Oil}}, \hat{X}_{t, 2030}^{\mathrm{Others}}$ are the predicted 2030 generation values at time $t$ for hydro, oil and other renewables, respectively, are assumed to be the same as their corresponding profile from 2015 in all three scenarios; $\hat{X}_{t, 2030}^{\mathrm{Coal}}$ is the predicted generation values at time $t$ of coal, which is assumed to be zero. These are summarized below.

$\hat{X}_{t, 2030}^{\text {Hydro }}=X_{t, 2015}^{\text {Hydro }}$

$\hat{X}_{t, 2030}^{\mathrm{Oil}}=X_{t, 2015}^{\mathrm{Oil}}$

$\hat{X}_{t, 2030}^{\text {Others }}=X_{t, 2015}^{\text {Others }}$

$\hat{X}_{t, 2030}^{\mathrm{Coal}}=0$

Section 2.3 describes the forecasting process for $\hat{X}_{t, 2030}^{\mathrm{WS}}$ in the three different scenarios, and corresponds to Step $2 b$ in Fig. 1.

\subsection{Predicting wind/solar generation in $\mathbf{2 0 3 0}$}

The historical wind/solar generation profile from 2009 to 2015 in New England is depicted below [27]. Our observation here is that as wind/solar penetration into the grid increases, so does the intermittency, or inter-day variability.

Because the time series appears to visually have a moving conditional mean and a moving conditional variance, an ARIMA-ARCH model (where ARIMA is an autoregressive integrated moving average process and $\mathrm{ARCH}$ is an autoregressive conditional heteroscedastic model) can be fitted to this time series to observe if it can predict and forecast this wind/solar generation time series. Previous works forecasting wind speed looks to ARMAARCH models [28, 29], further confirming this as a reasonable model of choice for wind generation, noting that wind generation is essentially proportional to wind speed. An ARMA model more generally is a linear stochastic model that has the form:

$$
\begin{aligned}
X_{t}^{\mathrm{WS}}= & \delta_{2}+\varphi_{1} X_{t-1}^{\mathrm{WS}}+\varphi_{2} X_{t-2}^{\mathrm{WS}}+\cdots+\varphi_{p} X_{t-p}^{\mathrm{WS}}+\varepsilon_{t} \\
& +\theta_{1} \varepsilon_{t-1}+\theta_{2} \varepsilon_{t-2}+\cdots+\theta_{q} \varepsilon_{t-q}
\end{aligned}
$$

where $\delta_{2}$ is the constant that is a function of the time series mean; $X_{t}^{\mathrm{WS}}$ is the current point or observation in the time series for wind/solar generation; $\varepsilon_{t}$ is the error term; $\varphi_{i}$ is the autoregressive parameter that weight the lagged $X_{t}^{\mathrm{WS}}$ values, $i=1,2, \ldots, p ; \theta_{i}$ are the moving average parameter that describes the lagged error term values, $i=1,2, \ldots, q$. The error term is a stochastic process that is normally distributed about a zero mean and a constant variance, $\varepsilon_{t} \sim N\left(0, \sigma^{2}\right)$. Given the volatile nature of the wind/solar generation time series, in principle it can be modeled as an ARIMA-ARCH model. However, as our goal is to extrapolate these time-series from 2015 over a long horizon up to 2030 and beyond, we found that the ARMA-ARCH approach led to significant errors. Therefore, we utilize an AR(1) model, and estimate the mean and variance of each time series and their trends over years based on actual data. A visual examination of Fig. 2 shows that the variance of the series is increasing even as the mean is increasing over time. Breaking the total time series down into each of the seven individual years yields Fig. 3.

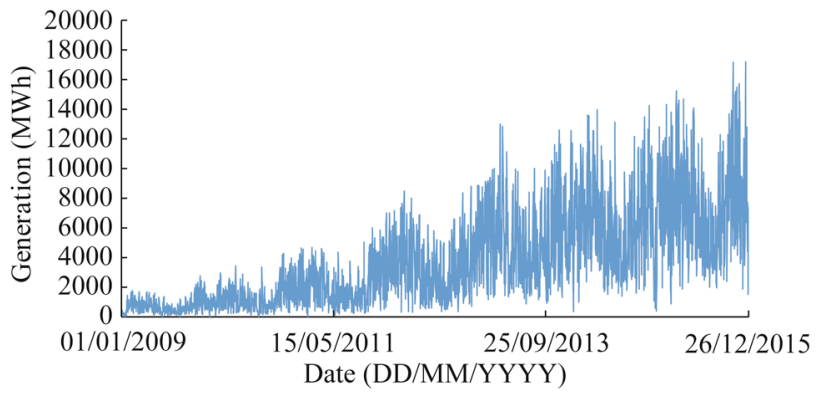

Fig. 2 Wind/solar actual daily generation from 2009 to 2015 in New England 


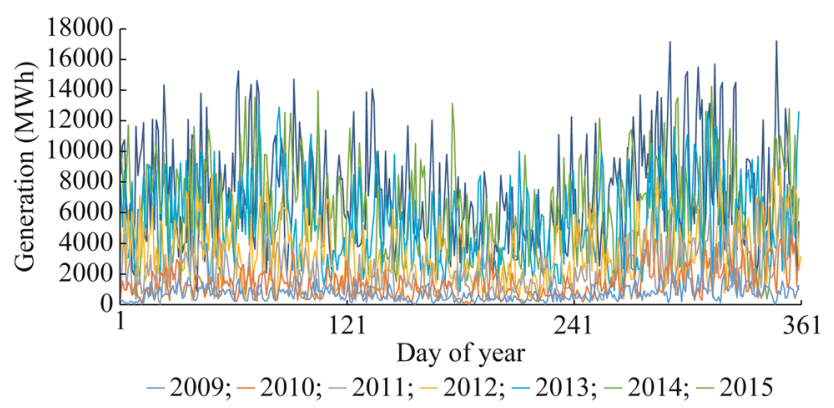

Fig. 3 Annual wind/solar generation profiles since 2009

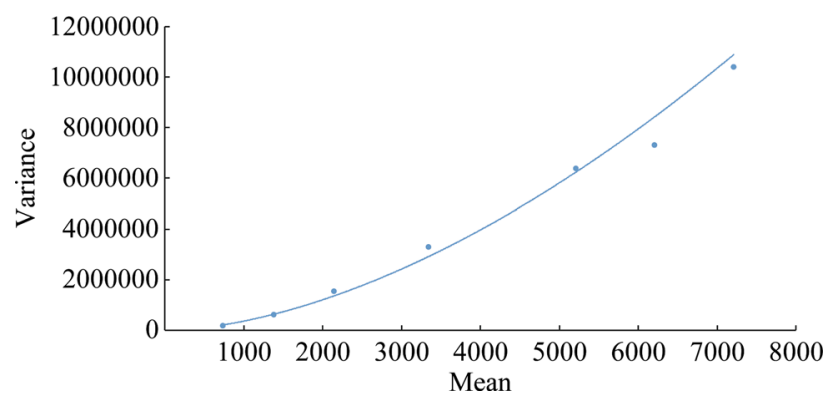

Fig. 4 Relationship between mean and variance on a yearly basis from 2009 to 2015

It appears that the yearly profile for wind/solar generation is similar year to year, but the mean and variance increases show similar trends. That is, every year the average generation seems to increase, and the volatility or variance between the daily points in each time series seems to increase as well. Specifically, the relationship between the mean $\mu$ and variance $\sigma^{2}$ of each yearly time series is portrayed in Fig. 4 below.This mapping between mean and variance is described by:

$\sigma^{2}=2.7299(\mu)^{1.7109}$

Hence, to create the wind/solar time series in 2030 given that it will encompass $10 \%, 20 \%$, and $30 \%$ of the generation mix, the variance is predicted given the mean inputs. According to the mean and variance mapping above, the variance is subsequently computed from their corresponding mean values in the three different scenarios.

To create the daily individual time series points, an autoregressive $\mathrm{AR}(1)$ process is fitted to the wind/solar generation time series from 2015, and the variance of the error term is calculated from (9). $X_{t}^{\mathrm{WS}}$ is assumed to be of the form:

$X_{t}=a+b X_{t-1}+\varepsilon_{t}$

Assuming that the error term $\varepsilon_{t}$ has a Gaussian distribution with zero mean and standard deviation sigma, the MLE method can be used to determine $a, b, \sigma$

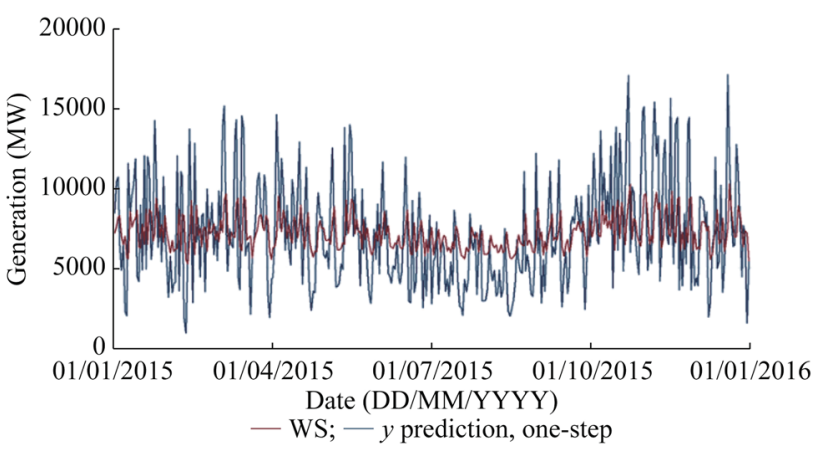

Fig. 5 Actual wind/solar generation in 2015 and predicted time series given an $\mathrm{AR}(1)$ model

from the time-series data. MLE is the method of fitting parameters to model a series of observations that maximize the likelihood of those observations given the parameters. It was found that for the wind/solar generation time-series in 2015, $a=7208, b=0.31, \sigma=3225$. The resulting AR (1) model in (10) is plotted in Fig. 5 and compared with the actual wind/solar generation for the 2015 time-series. The results illustrate that the AR model is successful in minimizing the phase error, but not the magnitude error. This in turn implies that the errors in the estimationof $a$ and $b$ are small whereas the estimate of the variance is significantly smaller than that of the actual time-series.

Given that the variance of the wind/solar generation is dependent on many factors that may be unknown or uncertain, such as how much capacity there is or what programs or incentives are in place, such an under estimation of the variance using our model may be reasonable. In addition, it can be argued that at its worst, our model provides an underestimation of the volatility in wind/solar generation.

With the AR(1) model as in (10), we now predict $\hat{X}_{t, 2030}^{\mathrm{WS}}$ using (9) and (10), for each of the three scenarios in Table 3 as follows. We begin with the mean $\mu$ of $\hat{X}_{t}^{\text {ws }}$ which is computed in 2015 as $\mu=7210.1$. The mean of the time series in each 2030 scenario equals the generation that would encompass $10 \%, 20 \%, 30 \%$ of the generation mix. That is, in the first scenario in which wind/solar encompasses on average $10 \%$ of the generation mix, the mean is determined as four times the 2015 mean, as the wind

Table 3 AR(1) models for wind/solar generation per scenario

\begin{tabular}{lll}
\hline Scenario & $\mathrm{AR}(1)$ model & Error term \\
\hline 2015 & $X_{t}^{\mathrm{WS}}=7208.3+0.31 X_{t-1}^{\mathrm{WS}}+\varepsilon_{t}$ & $\varepsilon_{t} \sim N(0,3224)$ \\
Scenario 1 & $\hat{X}_{t}^{\mathrm{WS} 1}=21,735+0.31 \hat{X}_{t-1}^{\mathrm{WS} 1}+\varepsilon_{t}$ & $\varepsilon_{t} \sim N(0,11487)$ \\
Scenario 2 & $\hat{X}_{t}^{\mathrm{WS} 2}=43,470+0.31 \hat{X}_{t-1}^{\mathrm{WS} 2}+\varepsilon_{t}$ & $\varepsilon_{t} \sim N(0,21070)$ \\
Scenario 3 & $\hat{X}_{t}^{\mathrm{WS} 3}=65,205+0.31 \hat{X}_{t-1}^{\mathrm{WS} 3}+\varepsilon_{t}$ & $\varepsilon_{t} \sim N(0,29806)$ \\
\hline
\end{tabular}




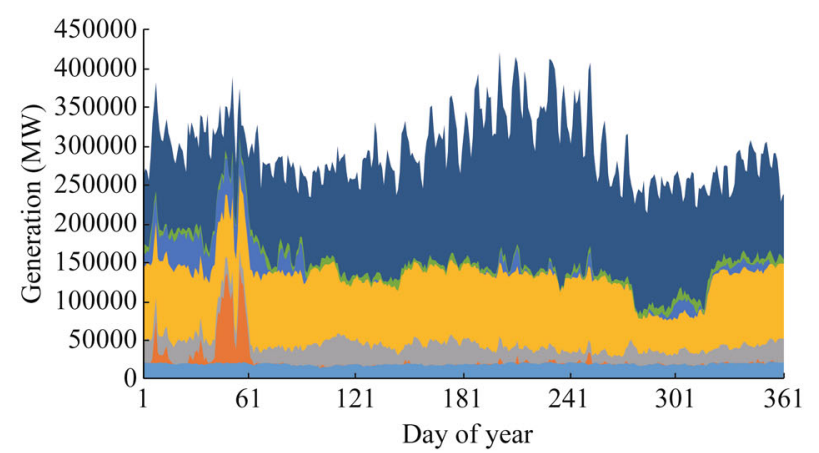

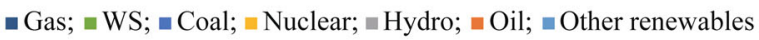

(a) Daily fuel generation mix breakdown 2015

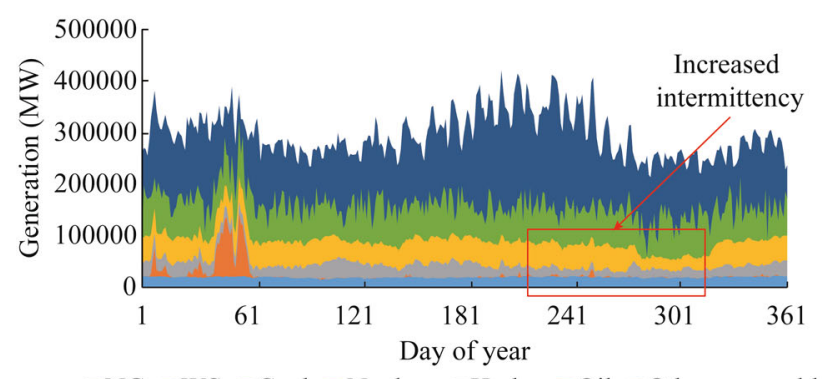

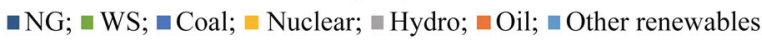

(c) Daily fuel generation mix breakdown in $2030 \mathrm{with}$ wind/solar at $20 \%$

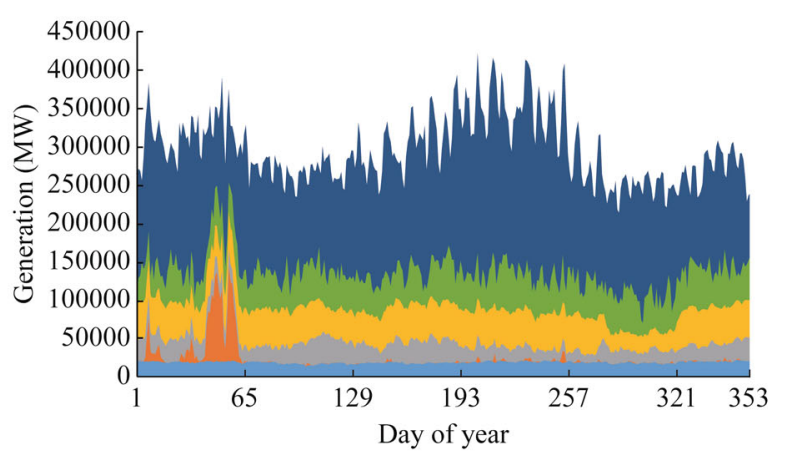

ఐ Gas; $₫$ WS; $₫$ Coal; $₫$ Nuclear; $₫$ Hydro; $₫$ Oil; $₫$ Other renewables

(b) Daily fuel generation mix breakdown in 2030 with wind/solar at $10 \%$

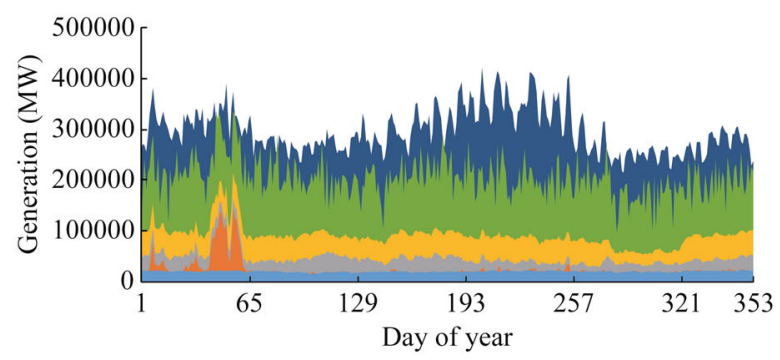

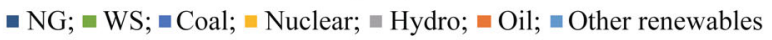

(d) Daily fuel generation mix breakdown in 2030 with wind/solar at $30 \%$

Fig. 6 Daily fuel generation mix in 2030 with wind/solar at 10\%, 20\%, 30\% of the generation mix, compared with that in 2015 (shown top-left)

generation in 2015 is about $2.5 \%$ of the overall generation mix. Similarly, in scenarios two and three, where wind/solar generation encompasses $20 \%$ and $30 \%$ of the generation mix, $\mu$ is $63000 \mathrm{MW}$ and $94500 \mathrm{MW}$, respectively, which corresponds to $700 \%$ and $1100 \%$ of the current proportion of wind and solar in the generation mix. Once $\mu$ is determined in these scenarios, the variance $\sigma^{2}$ can be determined from (9). Also, once the wind/solar generation is determined as in Table 3, (3)-(7) can be used to predict the amount of gas in the year 2030 for all three scenarios.

\subsection{A changing generation mix}

Assuming that AR parameter $b$ remains the same over the years, $a$ can be determined from (10) as:

$a=\mu(1-b)$

The assumption that $b$ remains the same implies that the time series correlation remains the same over the years, which is somewhat reasonable. The resulting time-series for all three scenarios together with the comparison with the 2015 series are summarized in Table 3. Once the wind/solar generation is determined as in Table 3, (3)-(7) can be used to predict the amount of gas in the year 2030 for all three scenarios, which is shown in Fig. 7.
The results shown in Fig. 6 represent one of the main results of this paper. That is, given greater wind and solar penetration in the grid, the intermittency or variability of gas increases on a daily basis. It should be noted that this intermittency is predicted by our model, even under the assumption of a constant electricity demand; if this demand increases as well, then the demand for gas could increase even further, causing further constraints on the gas pipeline infrastructure. These changes in wind/solar as well as gas generation profiles over the three scenarios will be used in a regression to determine the impact on gas price. The 2015 data of daily generation from each resource type was obtained from [27].

From Fig. 6 we can see that the wind/solar generation and gas generation become more variable. We compute a monthly volatility for wind/solar generation as [28]:

$$
\begin{aligned}
& X_{k}^{\mathrm{v}, \mathrm{WS}}=\sqrt{\frac{\sum_{t=1}^{N}\left(\Delta X_{t}^{\mathrm{WS}}-\Delta \bar{X}_{k}\right)^{2}}{(N-1) / N}} \\
& \Delta X_{t}^{\mathrm{WS}}=\ln \left(\frac{X_{t}^{\mathrm{WS}}}{X_{t-1}^{\mathrm{WS}}}\right) \\
& \Delta \bar{X}_{k}=\frac{1}{N} \sum_{t=1}^{N} \Delta X_{t, k}^{\mathrm{WS}}
\end{aligned}
$$

where $N$ is the number of the days in the month [30,31]. That is, to determine monthly volatility, we are calculating 
the monthly standard deviation over the daily relative change in value. Similarly, noting that the gas generation time series is determined using the wind/solar time series in Table 3 and (3), the corresponding annual volatility, $X_{y}^{\mathrm{v}, \mathrm{Gas}}$, can be determined as:

$X_{y}^{\mathrm{v}, \mathrm{Gas}}=\sqrt{\frac{\sum_{t=1}^{N_{t}}\left(\Delta X_{t}^{\mathrm{Gas}}-\Delta \bar{X}_{t}^{\mathrm{Gas}}\right)^{2}}{\left(N_{t}-1\right) / N_{t}}}$

where $N_{t}=365$, and $\Delta X_{t}$ is computed as in (13) and $\Delta \bar{X}_{t}$ is computed as in (14) but is an annual average over daily relative change. Table 4 shows the annual volatility of the gas generation in 2015 and each of the 2030 scenarios from Fig. 6.

Table 4 shows that when the wind/solar penetration increases to $10 \%$, the volatility metric more than triples, and rises to even greater levels with increasing penetration of wind and solar. With greater volatility in generation, there may be a number of implications for gas prices and the risk to GFGs in purchasing gas. We take a first step in the subsequent sections and use a regression model to explore some of these implications.

\subsection{Gas price regression analysis-three 2030 scenarios}

With the generation-mix profiles derived as in Step 1, and the wind-solar generation predicted as in Step 2, we now carry out a regression analysis to determine the relationship between the outcome variable of interest, which is the gas price paid by GFGs, denoted as $P_{k}^{\mathrm{Gas}}$, and the explanatory variables it could depend on. Our focus is on determining this relationship using monthly data over 2009 to 2015 as wind/solar generation started rising in 2009 in New England's generation mix. We identify these variables to be $X_{k}^{\mathrm{v}, \mathrm{WS}}$, the volatility in wind and solar generation, $X_{k}^{\mathrm{Gas}}$, gas generation, $X_{k}^{\text {Storage }}$, gas storage, and $X_{k}^{\mathrm{HHspot}}$, the Henry Hub spot price. The price $P_{k}^{\mathrm{Gas}}$ was assumed to be the same as the MA gas price charged to electricity consumers [32], due to lack of data for the other states in New England. $X_{k}^{\mathrm{v}, \mathrm{WS}}$ is the average monthly series as was determined as in (14) using the data and procedure outlined in Section 2.4. $X_{k}^{\mathrm{Gas}}$ is the average monthly power

Table 4 Volatility of NG in 2030 generation mix scenarios

\begin{tabular}{lc}
\hline Scenario & Volatility $\left(X_{k}^{\mathrm{v}, \text { Gas }}\right)$ \\
\hline 2015 & 0.9 \\
Scenario 1 & 3.1 \\
Scenario 2 & 5.6 \\
Scenario 3 & 16.6 \\
\hline
\end{tabular}

generated due to gas and is assumed to be proportional to the gas available to GFGs on the spot market. Data from [27] and (14) was used to construct $X_{k}^{\text {Gas }}$. Storage levels are considered as a source of supply, and because of our lack of granular data for MA storage levels, we used NY storage levels from EIA in [33] to determine $X_{k}^{\text {Storage }}$ assuming that they are similar, as geographically MA and NY experience similar weather and may therefore see similar injection patterns. Lastly, we include Henry Hub price from the EIA as that is the hub that determines gas prices elsewhere [34] to determine $X_{k}^{\mathrm{HHspot}}$. All of the above yielded 84 data points for each of the four time-series.

For the regression, we standardize each of the four variables as $x=(X-\mu) / \sigma$ where $x$ is the standardized variable corresponding to $X$ and $\mu$ and $\sigma$ are the mean and standard deviation, respectively, of the 84 data points over 2009 to 2015. The resulting regression model is of the form:

$P_{k}^{\mathrm{Gas}}=\alpha+\beta_{1} x_{k}^{\mathrm{v}, \mathrm{WS}}+\beta_{2} x_{k}^{\mathrm{Gas}}+\beta_{3} x_{k}^{\mathrm{HHspot}}+\beta_{4} x_{k}^{\text {Storage }}$

The regression model was fitted to actual New England or MA data from 2009 to 2015. The parameters $\alpha$ and $\beta_{i}$ were then identified via a sum of least squares regression method and led to:

$$
\begin{aligned}
P_{k}^{\mathrm{Gas}}= & 5.28+0.90 x_{k}^{\mathrm{v}, \mathrm{WS}}-1.37 x_{k}^{\mathrm{Gas}}+1.22 x_{k}^{\mathrm{HHspot}} \\
& -0.09 x_{k}^{\text {Storage }}
\end{aligned}
$$

Equation (17) shows that Henry Hub spot price and the gas generation explanatory variables have the largest impact on gas price, which is not surprising. Also, gas generation is inversely correlated with the gas price simply because it is a reflection of the amount of gas obtained from the gas network, with low deliveries during winter and higher price, and high deliveries during summer and lower price. That is, the inverse correlation of -1.37 is due to the fact that GFGs purchase leftover gas from the spot market and therefore prices are high in the winter with deliveries remaining low. Implicitly, this accommodates the congestion effects of the gas transmission network, even though we have not explicitly analyzed the underlying physical network and pipeline constraints. Endogeneity between price and generation is not accounted for in the regression analysis. That is, impacts of gas spot price on any of the explanatory variables are not identified via instrumentation.

The next dominant variable in our regression model is the volatility in wind/solar generation $x_{k}^{\mathrm{v}, \mathrm{ws}}$, which includes a regression coefficient of 0.90 . As will be shown later in Fig. 8, gas prices spike in later years when significant wind/solar generation rises but gas generation stays 
relatively the same, indicating that wind/solar generation can impact gas price; this is one of the main points of this paper. This effect is discussed in detail in the next section. The last term in (17) shows that storage effects are not that dominant.

In Section 2.6, this regression model will be used in forecasting gas prices given calculated and forecasted explanatory variables.

\subsection{Prediction of gas price in 2030 using regression model}

From the regression model in (17) we now predict the gas price $\hat{P}_{k, 2030}^{\mathrm{Gas}, i}$, corresponding to each of the three 2030 scenarios. First, we calculate the volatility in wind/solar from (3) and (12) and gas generation from (3), also shown in Fig. 6. This gives us $\hat{x}_{k}^{\text {v,WS }}$ and $\hat{x}_{k}^{\text {Gas }}$ Henry Hub spot price, $x_{k}^{\mathrm{HHspot}}$, is assumed to increase from on average about $\$ 2.63$ in 2015 to on average $\$ 5$ in 2030. This is based on an EIA forecast in [35] which predicts similar increases over the next decade in the Henry Hub price. We do not change storage levels because, even though there are pipeline projects under construction, it is under much debate on how much additional capacity is necessary [36]. In addition, line pack operates at its maximum and there is very little of it in New England that can be used as additional supply in times of high constraints and peak demand. Also, MA has been focusing on energy efficiency initiatives and may not require additional pipelines [37]. These resulted in the following computational model for the gas price for Scenario 1 .

$$
\begin{aligned}
\hat{P}_{k}^{\mathrm{Gas}}= & 5.28+0.90 \hat{x}_{k}^{\mathrm{v}, \mathrm{WS}}-1.37 \hat{x}_{k}^{\mathrm{Gas}}+1.22 \hat{x}_{k}^{\mathrm{HHspot}} \\
& -0.09 x_{k}^{\text {Storage }}
\end{aligned}
$$

Similar regression models can be derived for Scenarios 2 and 3 as well. Details of how these predicted quantities were calculated are shown in Appendix A.

Figure 7 shows $\hat{P}_{k, 2030}^{\mathrm{Gas}}$ for each scenario where $k$ is the observation on a monthly basis. We compare these prices with the average gas-price profile over the years 2009 2015. From (18), we can make the following important observation: when wind/solar generation accounts for on average $10 \%, 20 \%$ or $30 \%$ of the generation mix, the annual average gas price decreases by $29 \%$ in Scenario 1, and increases by $10 \%$ and $31 \%$ in Scenarios 2 and 3 , respectively. The average gas price paid by electric consumers in MA between 2009 and 2015 was $5.72 \$ /$ tcf. This drops to $\$ 4.05$ in scenario 1, but increases on average to $\$ 6.31$ and $\$ 7.49$ in Scenarios 2 and 3, respectively.

The next observation that we make from Fig. 8 is that the predicted gas prices do not follow the seasonal trends

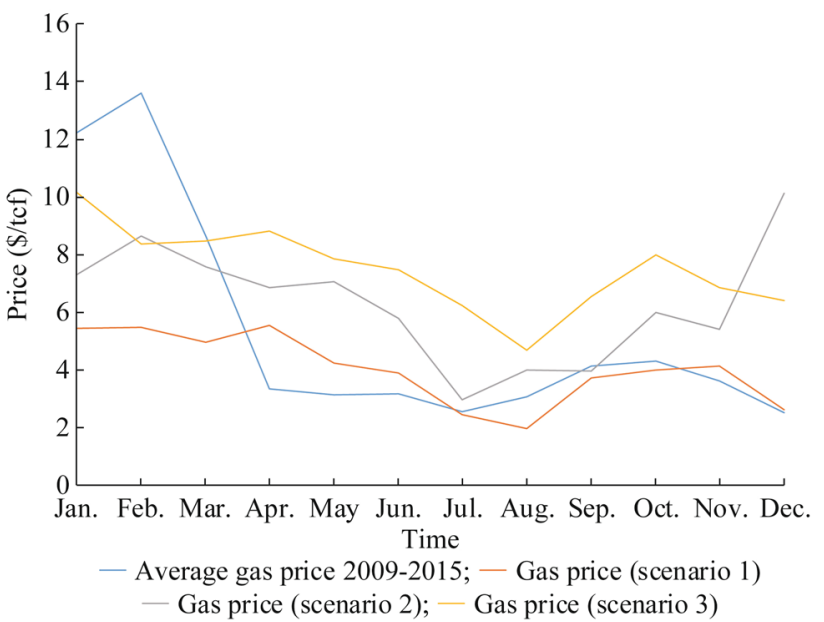

Fig. 7 Average gas prices from 2009 to 2015 and predicted gas prices in 2030 scenarios

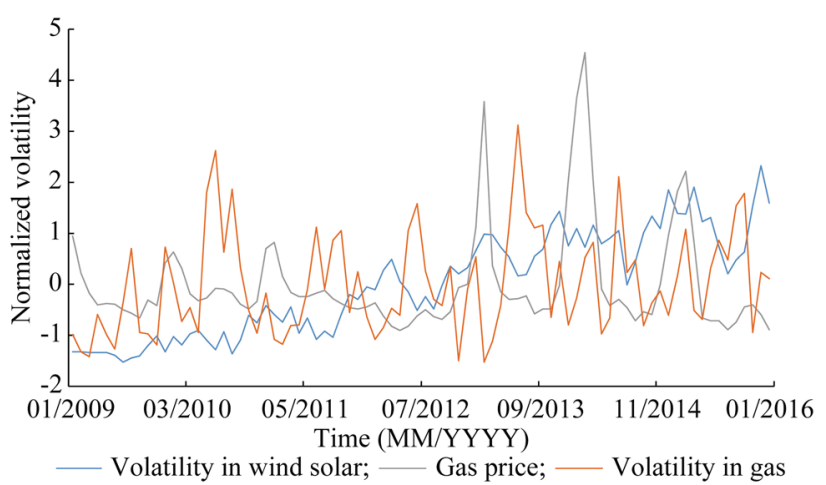

Fig. 8 Normalized values of volatility in wind/solar generation, volatility in gas generation, and gas price from 2009 to 2015

observed over 2009 2015. This could be because of two reasons: (1) we do not include pipeline congestion factors, which are more likely to occur in winter; (2) we do not take into account fluctuations in gas demand for heating. Both of these reasons can cause prices to increase in winter due to scarcity of gas and to fall in the summer due to abundance of gas. Neither of these factors were included in our model since we had no data related to MA pipeline constraints and storage and therefore could not perform a regression analysis with these elements. With more accurate and granular data, we could get a more significant relationship between these supply levels, leading to a better regression model and a gas price which could reflect more accurate patterns.

The decrease in gas price for Scenario 1 may be due to the fact that the effect of the decrease in gas generation overcomes the effect of increase in volatility. As we go towards Scenario 2, the latter effect may be stronger than the former, contributing to an increase; this trend continues as we move towards Scenario 3 . 
We also note that we only account for generation here. Even though less gas may be generated for the grid, with greater intermittent capacity coming online, more reliable capacity, or flexible gas capacity, will need to be available in times of great intermittency. This can be explained as follows. On a particular day, if wind/solar based generation is not available, and GFG is required, as this is the low cost flexible fuel, then GFGs need to be ready to make up the difference. Therefore, with greater wind/solar capacity committed to the grid, greater gas capacity or supply needs to be committed as well. This can also be corroborated by the current data from 2009 2015, shown in Fig. 8. While the variability in wind/solar increased, the variability in gas generation fluctuated within a particular range; however, gas prices spiked significantly over the years as wind/solar generation increased. Our model in (18) essentially implies that, if pipeline construction continues to lag behind wind/solar build out $[38,39]$, then this trend could continue as we move towards 2030 with further increases in gas prices.

Section 3 discusses how changes in gas price can impact the gas bids of GFGs in the gas spot market.

\section{Optimal auction model to analyze GFG bids in NG markets}

The second objective of this paper is to analyze how increased levels of wind/solar penetration impact GFG bids in the gas spot markets. In particular, we will take results obtained in Section 2 regarding the changed generation mix in the three scenarios in Fig. 7 as well as results from the gas regression model and evaluate their impact on gas bids. An optimal auction model is used to simulate the bilateral transactions on the natural gas spot markets. Inputs into the model include gas generation in the three scenarios, gas capacity on the spot market, and valuation for gas (spark spread determined by the difference between electricity price and forecasted gas price from the regression model). The main output of interest is the daily bids for gas. Gas bids are examined when GFGs bid on the spot market.

\subsection{Problem formulation}

The three main inputs into the model are gas plant valuation, gas plant demand, and gas plant capacity. The gas plant valuation in this paper is the difference between electricity price and gas price. The actual monthly gas and electricity prices from 2015 were used to determine the monthly valuation, $v_{k, 2015}$ in (19). The gas price prediction from the regression relations in (18) in Section 2 are used to determine the valuation in (20).

$$
\begin{aligned}
& v_{k, 2015}= \begin{cases}P_{k, 2015}^{\mathrm{E}}-P_{k, 2015}^{\mathrm{Gas}} & \hat{P}_{k, 2015}^{\mathrm{Gas}}<P_{k, 2015}^{\mathrm{E}} \\
0 & \hat{\mathrm{P}}_{\mathrm{k}, 2015}^{\mathrm{Gas}} \geq P_{k, 2015}^{\mathrm{E}}\end{cases} \\
& v_{k, 2030}= \begin{cases}\hat{P}_{k, 2030}^{\mathrm{E}}-\hat{P}_{k, 2030}^{\mathrm{Gas}} & \hat{P}_{k, 2030}^{\mathrm{Gas}}<\hat{P}_{k, 2030}^{\mathrm{E}} \\
0 & \hat{P}_{k, 2030}^{\mathrm{Gas}} \geq \hat{P}_{k, 2030}^{\mathrm{E}}\end{cases}
\end{aligned}
$$

where $\hat{P}_{k, 2030}^{\mathrm{E}}$ is on average assumed to be the same as the profile of the electricity prices over 2009 to 2015 , but scaled down to maintain an average price of about $42 \$$ / MWh, instead of about 48 \$/MWh (the former was the average electricity price in 2015 while the latter was the average price between 2009 and 2015 in MA). We do not project increased levels of electricity prices, unlike the projected average increase of $10 \%$ to $31 \%$ in the second and third scenarios of the gas price. This is because greater wind and solar buildout as well as increased volatility in gas-based generation may lead to higher-cost gas plants not getting dispatched. Energy efficiency programs also contribute to possible reduction in electricity prices. These reductions can offset any increase in the electricity price that may keep pace with the projected increase in gas price from our results in Section 2.6.

These monthly valuations in (19) and (20) are converted into daily valuations $v_{t}$, which serve as inputs into the auction model. That is, the average valuation over a particular month is used as the valuation on every day of that month. To differentiate between the daily values within a month, as well as the valuation between the two bidders in the model, a random variable with a Normal distribution between 0 and 1 is subtracted from the valuation of each day. This accounts for any noise on a particular day that can account for variation in pricing and valuation. The second input, gas demand, comes from $X_{t, 2015}^{\mathrm{Gas}}$ and $\hat{X}_{t, 2030}^{\mathrm{Gas}}$ in (3) in each of the three scenarios. That is,

$$
\begin{aligned}
& d_{t, 2015}=X_{t, 2015}^{\mathrm{Gas}} \\
& d_{t, 2030}=\hat{X}_{t, 2030}^{\mathrm{Gas}}
\end{aligned}
$$

The third input into the auction model is the capacity available to the GFGs on the spot market and is determined by assuming GFGs have unequal access to the spot market. As mentioned in the introduction, consumers who hold firm contracts on the gas markets have first priority in their bids for gas as well as for any overtaking of gas on the secondary markets. On a daily basis, the SRCM values for each observation $t$ are determined by:

$$
\begin{aligned}
c_{t} & =C_{p, t}-\gamma_{t} D_{\mathrm{LDC}, t} \\
\gamma_{t} & =\frac{D_{\mathrm{LDC}, t}}{D_{\mathrm{LDC}, \overline{\text { season }}}}
\end{aligned}
$$




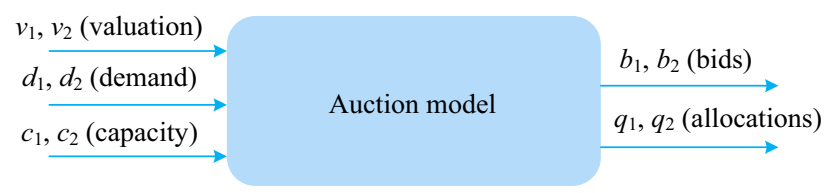

Fig. 9 Inputs and outputs from optimal auction model for natural gas markets considering two bidders

where $c_{t}$ is the capacity of the SRCM at $t ; C_{p, t}$ is the overall pipeline capacity at $t$, (algonquin pipeline through MA assumed in this problem formulation); $D_{\mathrm{LDC}, t}$ is the LDC demand at $t ; \gamma_{t}$ is the parameter representing the proportional fraction of how much an LDC may overtake from the secondary market. The data to compute the capacity $c_{t}$ was obtained from Algonquin deliveries into MA [40]. We maintain the daily capacities to be the same between 2015 and 2030 due to predictions of slow to no pipeline growth in the MA area [37].

Figure 9 depicts the inputs and outputs into the optimal auction model. The inputs and outputs show two each to represent the two bidders (GFGs) represented in our model. A mechanism design of an optimal auction model consists of a valuation structure for each bidder in the market where each bidder has a private value for gas at each day $t$. For these two bidders at a certain time $t$, their bidding values are denoted as $v_{1}$ and $v_{2}$. Each valuation is translated into a bid $b_{1}$ and $b_{2}$ based upon an optimal bidding strategy that is determined from a Bayesian-Nash equilibrium for the resulting game (transaction between gas marketer and GFGs).

To start, we examine the optimal auction from the perspective of the gas marketer where the gas marketer, or seller, aims to maximize his or her revenue when there are two bidders (GFGs). The gas is not considered a divisible good, but rather multiple indivisible goods where the "entire" good is given to the bidder with the highest valuation (their demand entirely met) and the next highest valuation satisfied with the remaining "second indivisible good" which is essentially the remaining gas [13, 41]. Once the bids $b_{i}$ are transmitted, the gas marketer determines how to allocate the gas to each player based upon their bids.

In order to determine the bidding procedure, we first compute the marginal revenue to the marketer who is the seller. If the seller offers the buyer a take-it-or-leave-it contract at a particular price of $x$, and $F(x)$ is the cumulative distribution function of $x$, then the probability that the buyer accepts this offer is $1-F(x)$, which represents the probability that the value for gas exceeds $x$. Hence if we set $x=v_{i}$, the valuation of gas, and interpret $v_{i}$ as the "price" then $q\left(v_{i}\right)=1-F\left(v_{i}\right)$ can be viewed as the demand curve, with the total revenue $R$ given by $v_{i} q\left(v_{i}\right)$. From the total revenue, one can determine $Q_{i}$, or the probability of allocation of gas, as the marginal revenue; that is, any buyer that is willing to pay higher has a higher probability of getting allocated their gas demands and will yield a higher marginal value to the gas seller. We therefore can compute the probability of allocation of gas $Q_{i}$ as:

$Q_{i}=\frac{d R}{d q}=v_{i}+q\left(v_{i}\right) \frac{d v_{i}}{d q}$

$Q_{i}=v_{i}-\frac{1-F\left(v_{i}\right)}{f\left(v_{i}\right)}$

where $f\left(v_{i}\right)$ is the corresponding density function [41]. That is, (26) implies that as $v_{i}$ increases, $Q_{i}$ increases as well.

Once the probability of allocation is determined as in (26), the actual allocation, $q_{i}$, is determined as follows. If bidder 1 yields the larger marginal revenue, he will get allocated the minimum of his entire demand, or total supply available, $S_{0}$. If bidder 2 yields the smaller marginal revenue, he gets whatever gas supply is left over. This is compactly expressed below in (25).

$q_{1}=\left\{\begin{array}{lll}\min \left(S_{\mathrm{o}}, d_{1}\right) & \text { if } & Q_{1}>Q_{2} \\ \max \left(0, S_{\mathrm{o}}-d_{2}\right) & \text { if } & Q_{1}<Q_{2}\end{array}\right.$

That is, if the bids are such that the corresponding $v_{i}$ leads to $Q_{i}$ greater than $Q_{j}$, then bidder $i$ gets full gas allocated.

However, marketers do not know the bidders' valuation function $v_{i}$ but only the bids $b_{i}$. We therefore pose the following maximization problem in order to determine the optimal bid, which in turn leads the bidder towards winning the auction.

$\max _{b_{i}} F\left(v_{i}\right)\left(v_{i}-b\right)$

The optimal bid needs to be such that the total cost that is being maximized is the product of the probability of getting the bidder $i$ 's gas demand met multiplied by the corresponding utility or payoff that bidder $i$ will get from that gas allocation. Suppose we define the bid to be a function of the valuation as $b_{i}=\beta\left(v_{i}\right)$, the first-order condition for this maximization problem in (28) is therefore given by:

$F(v) \beta^{\prime}(v)+f(v) \beta(v)=v f(v)$

The above ODE has the boundary condition, $\beta(0)=0$; the player does not bid if the valuation is 0 . With $f(v)=e^{-v}$, the following optimal bidding strategy is solved.

$b_{i}^{*}=\beta\left(v_{i}\right)=\frac{1-e^{-v_{i}}\left(v_{i}+1\right)}{1-e^{-v_{i}}}$

Such a bidding strategy corresponds to a Bayesian Nash Equilibrium in which there is an optimal strategy for 
bidders to bid into the market such that they gain no utility from deviating from that strategy [42].

In summary, the auction model above consists of a bidding strategy that GFGs can employ to signal to the gas marketer as to how much they value gas. Given these bids, the gas marketer determines, via the allocation rule $\left(Q_{i}\right)$, the probability of each bidder receiving gas and orders gas allocation assignments based on highest to lowest probability. From this, the allocation $q_{i}$ is assigned based on the bidder with the highest $Q_{i}$ receiving the minimum of total supply or all of his/her demand as described in (27), and the next highest bidder getting anything leftover.

\subsection{Results for GFG bidding volatility}

The auction model described in Section 3.1 assumed the same valuation for both bidders because both bidders were assigned the same price difference in electricity and gas prices. Therefore, to obtain slight variation between them, and also to account for any noise that can occur in prices, we simulated their valuation $v_{1}$ and $v_{2}$ on day $t$ by subtracting out a random variable between 0 and 1 from the valuation. The daily bids then calculated were a function of these valuation terms. From bids in (30), the annual volatility of the GFG bids is calculated as the standard deviation of the time series $b_{i}^{*}$ over 365 days. Table 5 shows the resulting volatility of GFG bids at present, when GFGs are on non-firm contracts in 2015. Also shown are the volatilities for the three 2030 scenarios discussed earlier.

The results in Table 5 clearly show that the volatility increase of GFG bids increases from 2015 gas bid volatility. It is interesting to note that as Wind/solar penetration increases from $20 \%$ to $30 \%$, the volatility increases by two orders of magnitude from 0.002 to 0.1 .

This in turn implies that with increasing penetration of renewables, there is greater uncertainty for GFGs to deliver and obtain gas and therefore they may be inclined to bid even less frequently, or have more secure hedges, in 2030 than in 2015. This is the main conclusion from Objective 2.

Table 5 Average volatility in GFG bids

\begin{tabular}{ll}
\hline Scenario & Volatility \\
\hline 2015 & $\sim 0$ \\
Scenario $1(2.5$ to $10 \%)$ & $\sim 0$ \\
Scenario $2(2.5$ to $20 \%)$ & 0.002 \\
Scenario $3(2.5$ to $30 \%)$ & 0.1 \\
\hline
\end{tabular}

\section{Summary and discussion}

This paper explored the electricity grid's greater reliance on natural gas through computational models depicting what the future markets may look like, and addressed two objectives:

1) To forecast and calculate future generation mix in 2030 for three scenarios of $10 \%$, and $20 \%$, and $30 \%$ average wind/solar generation, and to analyze how the associated volatility will affect volatility in gas generation and gas prices.

2) To analyze GFG bids in 2015 and 2030.

The main results of this paper are that with increasing wind/solar penetration: (1) volatility in gas generation increases; (2) gas prices go up on average, with a significant increase in the summer months and a slight decrease in the winter months; (3) volatility in gas bids increases. To expand further, in Result (1), we observed that the annual gas volatility increases rapidly from 1 to 16 , from 2015 to Scenario 3 in 2030 in Table 4, though the total gas generation goes down. In Result (2), for these scenarios of increased wind/solar volatility, no coal, and reduced nuclear, the gas spot prices are predicted to increase by $10 \% \sim 30 \%$ in Scenarios 2 and 3, compared to the 2009 2015 gas price average, or from approximately 5.72 \$/tcf to 6.31 \$/tcf in Scenario 2 and 7.49 \$/tcf in Scenario 3. To further evaluate the risk, the optimal auction model proposed lead to Result (3). In particular, as wind/solar generation increases from $20 \%$ to $30 \%$ of the total generation mix from 2015 to 2030, the risk or volatility in GFG bids increases by two orders of magnitude. The results reported here are predicated on a number of assumptions. Several other factors may change our conclusions. Increasing intermittent renewable generation and capacity the electric grid in MA causes risks in the way GFGs bid for gas. There has been much discussion on the role of gas in New England and its place within the grid. While it is undeniable that gas is the most reliable flexible fuel in times of intermittency, it might become costly if infrastructure cannot keep pace with demand. As discussed, while gas supply to the grid may decrease, additional gas capacity is necessary because there will be frequent occurrences when wind/solar cannot produce and gas has to step in in order to make up the greater difference. In order to facilitate this additional gas capacity, a suitable infrastructure needs to be in place to ensure smooth deliveries with minimal pipeline constraints; and to pay for this extra infrastructure, costs will have to be passed to electric rate payers and that could increase prices. Modeling of the capacity markets, however, is not addressed in this paper. 
Additional work will need to be done to account for tradeoffs in costs between different initiatives within the grid. That is, if additional pipelines are to be built, what is the additional cost of this pipeline versus securing reliability for the grid a different way? Would it still be worth paying high costs in the winter over securing firm contracts that can help build pipeline infrastructure?

Much greater pipeline infrastructure implies greater CAPEX that can be passed onto electric-ratepayers in the form of mandating firm contracts, etc. This is still up for contention and debate. There are different contracts GFGs could perhaps have that are not as expensive as firm contracts (but less expensive than what they would have to pay for continued non-firm contracts on the spot market). This could include deals between GFGs and renewable sources who rely on GFGs to produce for reliability. If wind/solar generators fail to produce according to the capacity market, they could use transactions with GFGs as a way to hedge their own penalties and help GFGs reliably pay for gas.

New initiatives and cost-cutting and energy saving measures have not been included here. For instance, given the recent impetus to pass a storage mandate in MA, storage in MA could contain $600 \sim 1766 \mathrm{MW}$ of energy by 2020 [43]. This can mean less volatility in wind/solar generation which would cause less volatility in gas generation. In this case, there would be more certainty in gas procurement and less need for supply with less need to alleviate congestion in the gas network. Technology use could be optimized based on lower CAPEX between gas pipelines and energy storage.

The results reported here can be viewed as a first step to link the volatility in wind/solar generation to prices, generation, and volatility in the natural gas based generation. We have obtained these results using simple regression models and an optimal auction model derived under a number of simplifying assumptions. Significant work remains to improve these models, relax the assumptions, and obtain more accurate predictions so as to better design a natural gas infrastructure that makes full use of a renewable-rich electricity infrastructure. In addition, while the scope of the paper has been on market models for the New England region in the United States, the same models can be used for other regions of the world as well. The exact results and conclusions however will depend on the weather, the structure of the gas market, and the availability of various assets that will complement renewables for power generation.

Acknowledgements This work was supported by National Science Foundation (NSF) initiative Award (No. EFRI-1441301). We would also like to acknowledge Christopher Knittel at MIT, Matt White and Eugene Litvinov at ISO-NE and Don Santa at INGAA for several useful discussions.
Open Access This article is distributed under the terms of the Creative Commons Attribution 4.0 International License (http:// creativecommons.org/licenses/by/4.0/), which permits unrestricted use, distribution, and reproduction in any medium, provided you give appropriate credit to the original author(s) and the source, provide a link to the Creative Commons license, and indicate if changes were made.

\section{Appendix A}

We show the explanatory variables in the prediction model (18) were derived. $d_{1}$ and $d_{t}$ are the first and last day of month $t$, with $N$ days. $\mu^{Z}$ and $\sigma^{Z}$ are mean and standard deviation of variable $z$.

Wind/solar volatility:

$$
\begin{aligned}
x_{t}^{\mathrm{v}, \mathrm{WS}} & =\frac{X_{t}^{\mathrm{v}, \mathrm{WS}}-\mu^{\mathrm{v}, \mathrm{WS}}}{\sigma^{\mathrm{v}, \mathrm{WS}}} \\
\hat{z}_{t}^{\mathrm{v}, \mathrm{WS}} & =\frac{1}{N} \sum_{k=d_{1}}^{k=d_{t}} \hat{X}_{k}^{\mathrm{v}, \mathrm{WS}} \\
\hat{x}_{t}^{\mathrm{v}, \mathrm{WS}} & =\frac{\hat{z}_{t}^{\mathrm{v}, \mathrm{WS}}-\mu^{\mathrm{v}, \mathrm{WS}}}{\sigma^{\mathrm{v}, \mathrm{WS}}}
\end{aligned}
$$

Gas generation:

$x_{t}^{\mathrm{Gas}}=\frac{X_{t}^{\mathrm{Gas}}-\mu^{\mathrm{Gas}}}{\sigma^{\text {gas }}}$

$\hat{z}_{t}^{\text {Gas }}=\frac{1}{N} \sum_{k=d_{1}}^{k=d_{t}} \hat{X}_{k}^{\text {Gas }}$

$\hat{x}_{t}^{\mathrm{Gas}}=\frac{\hat{z}_{t}^{\mathrm{Gas}}-\mu^{\mathrm{Gas}}}{\sigma^{\mathrm{Gas}}}$

Henry Hub spot price:

$\hat{z}_{t}^{\mathrm{HHspot}}=\left(z_{t}^{\mathrm{HHspot}}-\mu^{\mathrm{HHspot}}\right)+5$

where $\mu^{\mathrm{HH} \text { spot }}=2.63, \hat{x}_{t}^{\mathrm{HH} \text { spot }}=\frac{\hat{z}_{t}^{\mathrm{HH} s p o t}-\mu^{\mathrm{HHspot}}}{\sigma^{\mathrm{HH} p p o t}}$.

\section{References}

[1] Shahidepour M, Fu Y, Wiedman T (2005) Impact of natural gas infrastructure on electric power systems. Proc IEEE 93(5):1042-1056

[2] Jenkins S, Annaswamy A, Hansen J et al (2015) A dynamic model of the combined electricity and natural gas markets. In: Proceedings of the 2015 IEEE PES innovative smart grid technologies conference (ICGT'15), Washington, DC, USA, 18-20 Feb 2015, 5 pp

[3] Hobbs BF (1995) Optimization methods for electric utility resource planning. Eur J Oper Res 83(1):1-20 
[4] Renewable resource supply curve: Executive summary. White Paper. New England States Committee on Electricity (NESCOE) (2012)

[5] Forrest S, Macgill I (2013) Assessing the impact of wind generation on wholesale prices and generator dispatch in the Australian National Electricity Market. Energy Policy 59:120-132

[6] Gelabert L, Labandeirab X, Linaresc P (2011) An ex-post analysis of the effect of renewables and cogeneration on Spanish electricity prices. Energy Econ 33(S1):59-65

[7] Ketterer JC (2014) The impact of wind power generation on the electricity price in Germany. Energy Econ 44:270-280

[8] Mariner-Volpe B (1995) Trends in natural gas transportation rates. In: Energy policy act transportation study: interim report on natural gas flows and rates. DOE/EIA-0602(95). Energy Information Administration (EIA), Washington, DC, USA, pp 39-64

[9] Sullivan J (1988) The application of mathematical programming methods to oil and gas field development planning. Math Program 42(1):189-200

[10] Midthun KT (2007) Optimization models for liberalized natural gas markets. Norwegian University of Science and Technology, Trondheim

[11] Tomasgard A, Rømo F, Fodstad M et al (2007) Optimization models for the natural gas value Chain. In: Hasle G, Lie KA, Quak E (eds) Geometric modelling, numerical simulation, and optimization. Springer, Berlin, pp 521-558

[12] Hubbard R, Weiner RJ (1986) Regulation and long-term contracting in US natural gas markets. J Ind Econ 35(1):71-79

[13] Cremer L, Laffont JJ (2002) Competition in gas markets. Eur Econ Rev 46(4/5):928-935

[14] Cobanli O (2014) An application of cooperative game theory: Strategic investments in the natural gas network. Ph.D. Thesis. Humboldt University of Berlin, Berlin, Germany

[15] Gkonis P (2009) The LNG market: a game theory approach to competition in LNG shipping. Marit Econ Logist 11(2):227-246

[16] New England spot natural gas prices hit record levels this winter. Energy Information Administration (EIA), Washington, DC, USA (2014)

[17] Autoregressive models. In: Lesson 14: Time series \& autocorrelation. Pennsylvania State University, State College, PA, USA (2017)

[18] Massachusett's electricity future: Reducing reliance on natural gas through renewable energy. Union of Concerned Scientists (UCS), Cambridge, MA, USA (2016)

[19] New England 2030 power system study. ISO New England, Holoyoke, MA, USA (2010)

[20] New England generation fuel mix changes likely as Vermont Yankee nuclear plant retires. Energy Information Administration (EIA), Washington, DC, USA (2015)

[21] Conca J (2016) Natural gas: not renewables: is replacing nuclear. Forbes, 16 May 2016

[22] Dueñas P, Leung T, Gil M et al (2015) Gas-electricity coordination in competitive markets under renewable energy uncertainty. IEEE Trans Power Syst 30(1):123-131

[23] Renewable portfolio standards. National Renewable Energy Laboratory (NREL), Golden, Co, USA (2015)

[24] Wesoff E (2011) GE's gas-fired plants could enable more wind and solar power. Greentech Media (GTM), Boston, MA, USA

[25] Mohl B (2015) Forecast: electricity demand flat next 10 years. Common Wealth Magazine, 5 Nov 2015

[26] Charles RKM (2016) Regional estimates of the price elasticity of demand for natural gas in the United States. Ph.D. Thesis. Massachusetts Institute of Technology, Cambridge, MA, USA

[27] Operations reports: daily generation by fuel type. ISO New England, Holoyoke, MA, USA (2017)
[28] Gao S, He Y, Chen H (2009) Wind speed forecast for wind farms based on ARMA-ARCH model. In: Proceedings of the 1st international conference on sustainable power generation and supply, Nanjing, China, 6-7 April 2009, 4 pp

[29] Masseran N (2016) Modeling the fluctuations of wind speed data by considering their mean and volatility effects. Renew Sustain Energ Rev 54:777-784

[30] An analysis of price volatility in natural gas markets. Energy Information Administration (EIA), Washington, DC, USA (2007)

[31] Pindyck RS (2004) Volatility in natural gas and oil markets. J Energy Develop 30(1):1-20

[32] Massachusetts natural gas price sold to electric power consumers: Dollars per thousand cubic feet. Energy Information Administration (EIA), Washington, DC, USA (2017)

[33] Underground natural gas storage by all operators: million cubic feet. Information Administration (EIA), Washington, DC, USA (2017)

[34] Charles A, Broxson B, Peterson S (2006) Understanding natural gas markets. Spectra Energy Corp, Houston, MA, USA

[35] Annual energy outlook 2017. Energy Information Administration (EIA), Washington, DC, USA (2017)

[36] Knight P, Luckow P, Biewald B et al (2017) New England's shrinking need for natural gas. Synapse energy Economics Inc, Cambridge

[37] Healey M (2015) AG study: increased gas capacity not needed to meet state's electric reliability needs. The Official Website of the Attorney General of Massachusetts, 18 Nov 2015

[38] Reed M (2015) New England needs 2 new natural gas pipelines. Common Wealth Magazine, 16 April 2015

[39] Changing resources: How New England has responded to challenges associated with the expanded role of natural gas. ISO New England, Holoyoke, MA, USA (2016)

[40] Natural gas: data. Energy Information Administration (EIA), Washington, DC, USA (2017)

[41] Krishna V (2010) Auction theory, 2nd edn. Elsevier Science, San Diego

[42] Fudenberg T (2015) Game theory. Anne Books Pvt Ltd, New Delhi

[43] Spector J (2017) Massachusetts decided to set an energy storage target: what should it be?. Greentech Media (GTM), Boston

Neha NANDAKUMAR received the B.S. in Chemical Engineering and Engineering \& Public Policy from Carnegie Mellon University in 2013. She received the M.S. in Technology and Policy, Engineering Systems Division from MIT in 2016 where her research focused on the nexus between natural gas and electricity infrastructures. Currently she works for GE Capital Energy Financial Services as part of the Investment Strategy in Power group.

Anuradha M. ANNASWAMY received her Ph.D. in Electrical Engineering from Yale University in 1985. She has been a member of the faculty at Yale, Boston University, and MIT where currently she is the director of the Active-Adaptive Control Laboratory and a Senior Research Scientist in the Department of Mechanical Engineering. Her research interests pertain to adaptive control theory and applications to aerospace, automotive, and propulsion systems, cyber physical systems science, and CPS applications to Smart Grids, Smart Cities, and Smart Infrastructures. She is the author of a graduate textbook on adaptive control (2004), co-editor of two reports, IEEE Vision for Smart Grid Control: 2030 and Beyond," 2013, and Impact of Control Technology, 2014(2nd edition), and coauthor of over a hundred journal publications and numerous conference publications. She has received several awards including the George Axelby and 
Control Systems Magazine best paper awards from the IEEE Control Systems Society (CSS), the Presidential Young Investigator award from NSF, the Hans Fisher Senior Fellowship from the Institute for Advanced Study at the Technische Universität München in 2008, and the Donald Groen Julius Prize for 2008 from the Institute of Mechanical Engineers. She is a Fellow of the IEEE and IFAC. She served as the Vice President for Conference Activities (2014-15), and will serve as the VP for Technical Activities (2017-18) in the Executive Committee of the IEEE CSS. She has been both a nominated member (1993) and elected member of the CSS Board of Governors (2010-2012). 